\title{
Katholische Missionszeitschriften in den USA
}

\author{
von Lothar Janek
}

„Die Tage der Missionszeitschrift sind vorbei. Einige werden noch für eine Reihe von Jahren fortbestehen, weil ihre Verleger harte Verkaufsmethoden einsetzen. Das: sichert genügend Geld, um die Zeitschrift am Leben zu erhalten, garantiert aber keine Leser. Wenn wir heute noch Leute über die Missionssituation aufklären wollen, müssen wir für die weltliche Presse schreiben. Das setzt voraus, daß wir etwas zu sagen haben, was sich zu lesen lohnt, und daß wir es interessant und sinnvoll sagen. Wir müssen auch über das geschriebene Wort hinausgehen und unsere Botschaft mit Hilfe der ,kühlen Medien "verbreiten." - "Jede Kongregation und jede Missionsgesellschaft sollte $\mathrm{zu}$ ihrer eigenen Identifikation und zu einer wirksamen Werbung ihre eigene Zeitschrift haben. ... Wir werden niemals ohne unsere Zeitschrift sein.“

Diese zwei Zitate zeigen, wie unterschiedlich Redakteure amerikanischer Missionszeitschriften die Situation dieses besonderen Zweiges der kirchlichen Presse beurteilen.

„Können katholische Missionszeitschriften in den USA überleben?", das war die zentrale Frage der Magister-Arbeit des Verfassers. Die Arbeit mit dem Titel "Catholic Foreign Mission Magazines 1968. On the Way to Extinction?" wurde Ende 1969 begonnen und im Dezember 1970 von der Syracuse University, Syracuse, New York, angenommen. Um herauszufinden, ob katholische Missionszeitschriften eine Zukunft haben, wurde versucht, die gegenwärtige Lage dieser Publikationen herauszustellen und die wichtigsten Entwidklungen zwischen 1958 und 1968 aufzuzeigen. Nachträglich wurde die Studie beschränkt auf Zeitschriften, die von Ordensgemeinschaften herausgegeben wurden, die selbst Missionare ins Ausland schicken („mission-sending societies"). Da es kein Verzeichnis solcher Zeitschriften gab, wurden alle Orden angeschrieben, die 1968 wenigstens 50 amerikanische Mitglieder in den Missionen hatten oder bei denen auf je zehn Mitglieder in den USA wenigstens ein amerikanisches Mitglied kam, das außerhalb der Vereinigten Staaten als Missionar arbeitete. Die Umfrage erbrachte 35 Namen von Zeitschriften, die wenigstens zeitweise zwischen 1958 und 1968 erschienen waren.

Eine zweite Einschränkung war mit der Methode der Datensammlung gegeben: je ein Redakteur jeder dieser 35 Zeitschriften wurde gebeten, einen vom Verfasser erarbeiteten Fragebogen auszufüllen. Zweiunddreißig Schriftleiter gingen auf diese Bitte ein und beantworteten den Fragebogen wenigstens teilweise. Von den 32 Zeitschriften, die sie vertraten, existierten 18 noch Ende 1968, drei von ihnen gingen 1969 ein. Ende 1958 wurden schon 28 dieser 32 Zeitschriften verlegt, vier weitere wurden später gegründet.

Sieben der 18 Zeitschriften, die Ende 1968 herausgegeben wurden, erschienen zweimonatlich, drei vierteljährlich, drei andere elfmal im Jahr; die restlichen fünf erschienen monatlich bzw. fünf-, neun- und zehnmal im Jahr. Ungefähr ein Drittel aller Zeitschriften hat die Erscheinungsweise zwischen 1958 und 1968 geändert. Einige wechselten zu häufigerer Erscheinungsweise, um durch häufigeren Kontakt

P. Lothar Janek M. A. svd hat nach seiner theologischen Ausbildung in Deutschland und in den USA Publizistik studiert. Er ist heute Redakteur der von den Steyler Missionaren herausgegebenen Familienzeitschrift „Stadt Gottes“. 
mit den Lesern eine bessere Wirkung zu erreichen. Mehr Zeitschriften schränkten ihr Erscheinen jedoch ein; finanzielle Gründe wurden für diese Änderungen angegeben.

Die Auflagen der einzelnen Hefte waren sehr unterschiedlich, sie lagen 1968 zwischen 5000 und „über einer Million"; doch nur etwa 34 Prozent der Zeitschriften hatten Auflagen über 25000.

$\mathrm{Da}$ für einige Publikationen keine genauen Angaben zu erhalten waren, war es auch nicht möglich, die Gesamtauflage der Missionszeitschriften auszurechnen. Nach den vorliegenden, zum Teil sehr ungenauen Zahlen, lag sie 1958 bei „über“ 1248556 und 1968 bei „über “ 2314637 . Das „über “ könnte bedeuten, daß die tatsächliche Auflage in beiden Fällen um eine halbe Million höher lag. Jedenfalls war die Gesamtauflage trotz der geringeren Zahl der Blätter gewachsen, und dieses Wachstum ging nicht nur auf das Konto einiger Magazine mit sehr hohen Auflagen und Auflagengewinnen.

Um die Höhe der Auflagen recht zu bewerten, muß man allerdings auf den Vertrieb der Zeitschriften achten. Nur sechs von 17 Heften, die noch Ende 1968 erschienen, wurden hauptsächlich an Abonnenten verschickt; neun gingen vor allem an Leute, die etwas für die Mission gespendet hatten. Wenigstens acht Orden sandten ihr Blatt zu jedem, der eine Geldspende gemacht hatte, die wenigstens so hoch war wie das Abonnement ihrer Zeitschrift. Nach Schätzungen von 13 Redakteuren wurden ungefähr 83 Prozent der Gesamtauflage ihrer Zeitschriften zum Dank für Spenden verschickt. Auch die Auflagensteigerungen zwischen 1958 und 1968 gingen größtenteils darauf zurück, daß die Blätter an mehr Leute verschickt wurden, die die Missionsarbeit finanziell unterstützten.

Missionszeitschriften galten immer als ein wichtiges Mittel der Werbung, und Werbung für die Mission war oft verbunden mit einer Attacke auf den Geldbeutel. Deshalb wurden Missionszeitschriften in den Vereinigten Staaten gelegentlich als

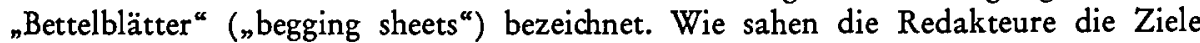
ihrer Zeitschriften?

Der Fragebogen nannte sieben mögliche Ziele einer Missionszeitschrift; der Redakteur wurde gebeten, die Ziele seines eigenen Blattes herauszusuchen und sie nach ihrer praktischen Bedeutung für die Redaktionsarbeit zu ordnen. Weitere Ziele konnten hinzugefügt werden. Die Antworten wurden in Zahlenwerte umgesetzt, und zwar so, daß sowohl die Häufigkeit, mit der ein Ziel genannt wurde, als auch die jeweilige Plazierung einberechnet wurden. Hätten alle Schriftleiter dieselben drei Ziele immer in derselben Reihenfolge aufgeführt, dann hätten sich 1,000 für das erste, 0,500 für das zweite und 0,333 für das dritte Ziel ergeben. Für 17 Zeitschriften, die noch Ende 1968 erschienen, ergaben sich die folgenden Werte: Missionserziehung 0,818, Information über den Orden 0,453, Berufswerbung 0,320, finanzielle Unterstützung 0,300, geistige Unterstützung 0,247, allgemeine religiöse Erziehung 0,057, Unterhaltung 0,027. Für elf Hefte, die schon vor 1968 eingestellt worden waren, waren die Werte ähnlich (Ziele in derselben Reihenfolge wie oben): 0,$806 ; 0,424 ; 0,324 ; 0,269 ; 0,136$; 0,030 .

$\mathrm{DaB}$ die Redakteure der Missionserziehung bei weitem die größte Bedeutung zumaßen, ist verständlich, war aber doch nicht ohne weiteres zu erwarten. Offenbar waren sich die Schriftleiter bewußt, daß ein Verständnis für die Missionsaufgabe und Information über die Missionstätigkeit eine fortdauernde Unterstützung der Mission gewährleistet. Es wäre allerdings falsch anzunehmen, daß die Mehrzahl der 
Redakteure die Missionserziehung unabhängig von der Tätigkeit des eigenen Ordens gesehen und gemeint hätte. Einige von ihnen hatten sich zwar in den letzten Jahren bemüht, ihre Leser mehr als früher auf die Missionspflicht und Missionsarbeit der ganzen Kirche hinzuweisen, andere aber haben sich auch 1969 noch darauf beschränkt, die Tätigkeit der eigenen Gesellschaft darzustellen. Schließlich waren alle hier untersuchten Missionszeitschriften auch Publikationen der Ordenspresse mit der Aufgabe, um Unterstützung für die Tätigkeit der einzelnen Gesellschaft zu werben. Man kann unter diesen Missionszeitschriften zwei Gruppen unterscheiden: solche, die den Hauptakzent auf den Orden legen, und die größere Zahl, die an erster Stelle die Missionstätigkeit (des Ordens) betont. Es ist nicht überraschend, daß „Information über den Orden" den zweithöchsten Punktwert erhielt.

Viele Redakteure sind überzeugt, daß sie zur Missionserziehung der Gläubigen viel beigetragen haben: 18 von 24 Schriftleitern zählten Missionserziehung zu den Gebieten, auf denen sie am erfolgreichsten waren. Zwölf Redakteure hielten Information über den Orden für ein Ziel, das sie mit am besten erreicht hatten. Freilich ist in beiden Fällen der Erfolg schwer zu messen.

Berufswerbung nimmt unter den Zielen den dritten Platz ein, doch nur fünf Schriftleiter betrachteten ihre Zeitschrift als erfolgreich auf diesem Gebiet, und einer dieser fünf beschränkte diesen Erfolg ausdrücklich auf die Vergangenheit, während zwei andere zu erkennen gaben, daß sie vom Erfolg in der Vergangenheit schlossen, daß ihr Blatt noch immer diese Aufgabe erfüllte. Acht Redakteure zählten Berufswerbung ausdrücklich zu den Zielen, die am wenigsten erreicht worden waren. Ein Unterschied ist hier festzustellen zwischen den Antworten von Heften, die noch existierten, und denen von eingegangenen Zeitschriften. Drei der Blätter, die noch Ende 1968 am Leben waren, rechneten Berufswerbung zu den Zielen, die am besten, sechs zählten sie zu denen, die am wenigsten erreicht worden waren. Von den Zeitschriften, die nicht mehr erschienen, wurde sie zweimal als erfolgreich und zweimal als wenig erfolgreich bezeichnet. Spiegelt sich darin das Absinken der Missionsberufe in den letzten Jahren?

Etwas überraschend mag es erscheinen, daß finanzielle Unterstützung der Missionstätigkeit nur den vierten Rang unter den Zielen der Missionszeitschriften einnahm; nur ein Redakteur hatte sie als erste Aufgabe seiner Publikation bezeichnet. Unterschiedlich wurde der Erfolg dieser Werbung von Blättern, die noch existierten, und denen, die eingestellt worden waren, beurteilt. Acht Schriftleiter von noch erscheinenden und nur $z$ wei von eingegangenen Zeitschriften berichteten, daß sie in dieser Werbung erfolgreich waren; drei Redakteure von bestehenden, jedoch fünf von nicht mehr erscheinenden Blättern gaben $z u, d a ß$ sie in der Werbung um finanzielle Unterstützung versagt hatten. Andererseits sollen aber je sechs Zeitschriften jeder der beiden Gruppen Ausgaben verursacht haben, die höher lagen als die Einnahmen aufgrund dieser Zeitschriften. Sieben von zehn Blättern, die Ende 1968 noch erschienen, sollen nur 5 Prozent oder weniger der Missionsgelder ihrer Gesellschaft eingebracht haben; auf je ein Heft wurden 6-10, 11-25 und 76 Prozent oder mehr der Missionsgelder eines Ordens zurückgeführt. Hefte, die schon früher eingestellt worden waren, hatten noch weniger erbracht. Besondere Spendenaufrufe in den Zeitschriften scheinen in den meisten Fällen auch nicht sehr erfolgreich gewesen zu sein.

Diese Angaben zeigen, daß im allgemeinen Missionszeitschriften nicht die großen Geldquellen waren, für die sie oft gehalten wurden. $\mathrm{Zu}$ den Aussagen der Redakteure muß allerdings vermerkt werden, daß es sich in vielen Fällen nur um Schätzungen 
handelt. $\mathrm{Da}$ Geld für verschiedene Zwecke oft an verschiedene Adressen geschickt wurde, in manchen Fällen direkt an einzelne Missionare, wußten manche Gesellschaften nicht, wieviel Geld überhaupt eingekommen war, und von Spenden, die bekannt waren, konnte in vielen Fällen nicht festgestellt werden, ob sie irgendwie auf die Zeitschrift zurüdkgingen oder nicht. Das macht eine zuverlässige Schätzung der finanziellen Bedeutung eines Blattes äußerst schwer, wenn nicht gar unmöglich. Deshalb hatten sich mehrere Redakteure auch geweigert, irgendwelche Schätzungen zu versuchen.

Geistige Unterstützung der Missionsarbeit wurde von einem Redakteur als Hauptziel seiner Zeitschrift angegeben, doch je sechs Schriftleiter von noch existierenden und von eingegangenen Blättern nennen sie überhaupt nicht als Ziel. Allgemeine religiöse Erziehung und Unterhaltung spielen schließlich eine vollkommen nebensächliche Rolle. Es wäre eine interessante Aufgabe, durch Inhaltsanalyse festzustellen, wieweit sich die von den Redakteuren angegebenen Ziele und ihre Rangordnung in der Gestaltung der einzelnen Zeitschriften auch fassen lassen.

Vierzehn Redakteure von Zeitschriften, die Ende 1968 noch erschienen, beantworteten die Frage nach den Hauptschwierigkeiten, mit denen sie zu kämpfen haben. Jeder zweite erwähnte den Mangel an (ausgebildetem) Personal; fünf nannten den Mangel an Material, vier finanzielle Schwierigkeiten, drei die Erhaltung oder Vergrößerung der Auflage, je zwei die Verständnislosigkeit der Oberen und die ungenügende Zahl an Schreibern und Korrespondenten, je einer fehlende Zusammenarbeit innerhalb der Gesellschaft und mangelndes Interesse der Katholiken an der Mission. Einige Antworten zu anderen Fragen können diese Aussagen näher erklären und einige Zusammenhänge $z$ wischen verschiedenen Schwierigkeiten aufzeigen.

Zehn von 16 Zeitschriften, die noch Ende 1968 erschienen, hatten nur einen Redakteur; in zwei Fällen war dieser nicht einmal für diese Arbeit freigestellt. Vier weitere Redaktionsstäbe bestanden aus je zwei Leuten, die übrigen zwei aus drei Schriftleitern. An fünf Zeitschriften arbeitete kein Redakteur mit einer fachlichen Ausbildung. Bei weitem die meisten Redakteure waren Mitglieder der Missionsgesellschaften.

Der Mangel an Material und eine ungenügende Zahl von Schreibern und Korrespondenten stellen dasselbe Problem von zwei Seiten dar. Angaben über regelmäßige Mitarbeiter in den Missionen sind sehr ungenau, doch sie deuten darauf hin, daß die meisten Zeitschriften nur wenige solcher Mitarbeiter hatten. Sieben von 16 Redakteuren haben niemals Artikel von freischaffenden Autoren gekauft, nur zwei haben es regelmäßig getan. Fünf von elf hatten überhaupt keine anderen Materialquellen als die Mitglieder ihrer Orden, vier benutzten Veröffentlichungen der eigenen Gesellschaft in anderen Ländern oder Provinzen oder Publikationen eines anderen Zweiges des Ordens, drei Redakteure verwerteten religiöse Nachrichtendienste und Zeitungen aus dem Ausland. Einige Redakteure haben sich wohl nicht um andere Quellen bemüht, weil sie grundsätzlich nur Material aus den eigenen Missionsgebieten veröffentlichten oder von Mitgliedern des Ordens reichlich mit Material versehen wurden.

Nur drei Redakteure vermerkten die Schwierigkeit, die Auflage ihrer Blätter zu erhalten oder zu vergrößern; tatsächlich ist dieses Problem aber fast allgemein. Zehn Redakteure von Zeitschriften, die Ende 1968 noch vertrieben wurden, beantworteten die Frage nach den Gründen, die die Auflage ihrer Blätter zwischen 1958 und 1968 
haben wachsen lassen; alle zehn nannten als Grund eine weitere Verteilung der Hefte an Leute, die etwas für die Mission gespendet hatten, nur ein Redakteur nannte außerdem auch eine erfolgreiche Werbung.

In den letzten Jahren ist die Werbung für Missionszeitschriften erheblich erschwert worden, da die beiden wichtigsten Methoden teilweise oder ganz ausfielen: Kanzelwerbung und Werbung durch Schüler der Pfarrschulen. Briefaktionen zur Gewinnung neuer Leser und der Einsatz von Zeitschriftenwerbern erwiesen sich im allgemeinen als wenig erfolgreich oder als zu teuer; Werbung durch Missionsvereine und andere Laiengruppen ging zurück.

Mangel an Personal, Autoren und Material gehen teilweise auf ungenügende Finanzierung der Blätter zurück, teilweise aber auch auf mangelhafte Unterstützung von Seiten des Ordens. Diese ungenügende Unterstützung hat verschiedene Gründe: Uberbelastung der Missionare, Unzufriedenheit mit der gegenwärtigen Zeitschrift, überspannte Forderungen an dieses Blatt aufgrund unrealistischer Vorstellungen dessen, was bei den gegebenen Umständen überhaupt möglich ist, ferner Interessenlosigkeit gegenüber Pressearbeit und Werbung, generelle Ablehnung einer Missionszeitschrift. Ein unsicher gewordenes Selbstverständnis der Orden, Unsicherheit über die eigentliche Aufgabe der eigenen Gesellschaft und der Missionstätigkeit und die Suche nach neuen Wegen und Methoden haben die weitverbreitete negative Haltung gegenüber Missionszeitschriften innerhalb der Orden, die sie herausgeben, mit verursacht.

Zeitschriften, die Ende 1968 noch erschienen, hatten grundsätzlich mit denselben Schwierigkeiten zu kämpfen, denen die anderen Blätter zum Opfer gefallen waren; sicher werden auch in den nächsten Jahren noch einige kleinere Blätter eingestellt werden. $\mathrm{Ob}$ aber die Tage der Missionszeitschriften der Orden allgemein gezählt sind, wird wesentlich davon abhängen, ob die Missionsgesellschaften, Obere wie andere Mitglieder, die Bedeutung ihrer Publikationen einsehen und sie großzügiger als bisher unterstützen, oder ob sie weiterhin erwarten, mit einem Minimum an Personal, Geld und Arbeit erfolgreich für die Missionsidee und die Unterstützung ihrer Arbeit werben zu können. Eine Zusammenarbeit der verschiedenen Missionszeitschriften wäre empfehlenswert; sie könnte helfen, die Qualität einiger Blätter zu heben. Möglicherweise werden kleinere Zeitschriften nur überleben können, wenn sie sich zusammenschließen, doch ein Zusammenschluß wird von den meisten Redakteuren wegen der Schwierigkeit der Zusammenarbeit abgelehnt.

Letzten Endes wird natürlich die Zukunft der amerikanischen Missionszeitschriften vom Schicksal der Missionsgesellschaften und der Kirche in den Vereinigten Staaten abhängen, vor allem auch davon, ob sie ihre Kraft in der Lösung heimischer Probleme erschöpfen, oder ob sie sich von der offenbar wachsenden Introvertiertheit abwenden und wieder stärker den Problemen der Weltkirche zuwenden.

Folgende Zeitschriften wurden befragt:

AFRICA. Piscataway, N. J. Missionary Sisters of Our Lady of Africa. Eingestellt 1969.

AFRICAN ANGELUS. Tenafly, N. J. African Mission Fathers. Eingestellt 1963.

AFRICAN YOUTH. St. Paul, Minn. Sisters of St. Peter Claver.

CATHOLIC LIFE. Detroit, Mich. PIME Missionaries.

COLUMBAN FATHERS MISSIONS. St. Columbans, Neb. Columban Fathers.

CONSOLATA MISSIONS. Somerset, N. J, Consolata Mission Society. 
DIVINE WORD MISSIONARIES. Techny, Ill. Divine Word Missionaries.

DOMINICAN MISSION IMPACT. Washington, D. C. St. Jude Dominican Missions. Eingestellt 1967.

ECHO FROM AFRICA. St. Paul, Minn. Sisters of St. Peter Claver.

FRIARS' FIELDS. Hoboken, N. J. Conventual Franciscan Missions. Eingestellt 1966.

FRONTIERS FOR CHRIST. Arlington, Va. The Immaculate Heart Missions. Eingestellt 1962.

HOLY CROSS MISSIONS. Washington, D. C. Holy Cross Foreign Mission Society. Eingestellt 1959.

(ISLES OF MARY. San Antonio, Texas. Missionary Oblates of Mary Immaculate, Philippine Province. Verschmolzen mit MARY IMMACULATE zu OMI MISSIONS.)

JESUIT MISSIONS. New York, N. Y. Jesuit Missions, Inc. Eingestellt 1967.

THE LIGHT. Chicago, Ill. S. V. D. Catholic Universities. Eingestellt 1967.

MARIST MISSIONS. Framingham Centre, Mass. Missionary Sisters of the Society of Mary, Inc. Eingestellt 1963.

(MARY IMMACULATE. San Antonio, Texas. Missionary Oblates of Mary Immaculate, Southern Province. Verschmolzen mit ISLES OF MARY zu OMI MISSIONS.)

MARYKNOLL. Maryknoll, N. Y. Catholic Foreign Mission Society of America.

THE MASTER'S WORK. Techny, Ill. Holy Spirit Missionary Sisters.

MEDICAL MISSIONARY. Philadelphia, Pa. Society of Catholic Medical Missionaries, Inc.

MISSION ECHOES. Albany, N. Y. Missionary Sisters of the Immaculate Heart. Eingestellt 1964.

MISSIONHURST. Arlington, Va. Immaculate Heart Missions.

MSC DIMENSIONS. Aurora, Ill. Society of the Missionaries of the Sacred Heart. Eingestellt 1969.

OBLATE MISSION NEWS. Hudson, N. H. Oblates of Mary Immaculate, St. John the Baptist Province. Eingestellt 1967.

THE OBLATE WORLD AND VOICE OF HOPE. Boston, Mass. The ObIate Fathers, Our Lady of Hope Province.

OMI MISSIONS. San Antonio, Texas. Vgl. ISLES OF MARY und MARY IMMACULATE.

PEOPLE OF GOD. North Providence, R. I. Franciscan Missionaries of Mary. Eingestellt 1966.

REDEMPTORIST MISSIONS. Liguori, Mo. Redemptorist Fathers. Eingestellt 1968.

SANDAL PRINTS. Detroit, Mich. Capuchin Friars. Die Zeitschrift besteht noch, ist jedoch keine Missionszeitschrift mehr.

VERONA FATHERS' MISSIONS. Cincinnati, Ohio. Verona Fathers.

WHITE FATHERS MAGAZINE. Washington, D. C. The White Fathers, Inc.

XAVERIAN MISSIONS. Franklin, Wis. Xaverian Missionary Fathers. Eingestellt 1969.

ZEAL. St. Bonaventure, N. Y. St. Elizabeth Mission Society of Sisters of St. Francis.

Von den folgenden Zeitschriften wurde der Fragebogen nicht zurückgesandt:

THE MILL HILL WORLD. Albany, N. Y. Mill Hill Missionaries.

MISSIONARY YOUTH. Techny, Ill. Divine Word Missionaries. Eingestellt 1967.

MISSION DIGEST. Fraser, Mich. Xaverian Mission Sisters. Eingestellt $1967 ?$.

In dieser Liste ist der Name des jeweiligen Herausgebers in der Form wiedergegeben, die im Impressum der Zeitschrift gebraudht worden ist.

\section{S U M M A R Y}

Catbolic Foreign Mission Magazines 1968. On the Way to Extinction? This is the title of a master's thesis written at Syracuse University. The study tries to picture the situation of mission magazines in 1968 and important changes which had occurred since 1958. Data were collected by means of a questionnaire. It was returned by the editors of 32 (out of 35) magazines, published by mission-sending societies sometime between 1958 and 1968 . Some 
of the findings: Although the number of magazines declined from 28 in 1958 to 18 in 1968, the combined circulation increased greatly. Most magazines were distributed in response to donations; true subscribers played a secondary role. Mission education was generally considered the first goal of a magazine. Lack of (trained) personnel, material and writers, financial difficulties, little understanding of the importance of the periodical on the part of superiors and other members of the publishing orders, and insufficient cooperation within the individual society were the main problems in publishing the magazines. An increased cooperation between the smaller periodicals and wholehearted support by the publishing societies will be necessary to keep the mission magazines alive.

\section{R E S U M E N}

Catbolic Foreign Mission Magazines 1968. On the Way to Extinction? (Las Revistas Católicas sobre las Misiones Extranjeras 1968. En camino de extinción?). Tal es el título de una tésis doctoral presentada en la Syracuse University. Este estudio trata de describir la situación de las revistas misionales en 1968, y los cambios más importantes que se han verificado desde 1958. Los datos fueron conseguidos mediante cuestionarios enviados a las 35 Congregaciones misioneras que, entre los años 1958 y 1968, enviaban misioneros a países extranjeros y publicaban revistas misionales. 32 editores respondieron a los cuestionarios. Algunos resultados de esta encuesta: aunque el número de las revistas, entre 1958 y 1968 , disminuyó de 28 a 18, aumentó considerablemente el número de las tiradas. La mayoría de las revistas se enviaron a personas que contribuían con donativos a los trabajos misionales de dichas Congregaciones; los subscriptores fijos jugaban un papel secundario. La educación misional era considerada, generalmente, como el fin primordial de la revista. La falta de personal especializado, de material y escritores, las dificultades financieras, la poca comprensión de la importancia de la publicación de estas revistas por parte de los Superiores y otros miembros de las Ordenes publicadoras, así como la escasa colaboración dentro de las mismas Congregaciones editoras, fueron los problemas mayores en la publicación de estas revistas. Para que las revistas misionales puedan seguir publicándose, será necesario que aumente la colaboración entre las revistas de menor tirada y el decidido apoyo de las Congregaciones editoras. 